\title{
Marcando la cancha: una aproximación al fútbol femenino desde las ciencias sociales
}

\# XXCTG . KNG / CTVі̀

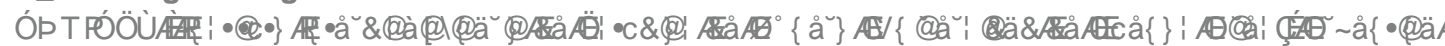

GPQY GN JQOAKECO

Cita sugerida: Alvarez Litke, M. (2018). Marcando la cancha: una aproximación al fútbol femenino desde las ciencias sociales. Cuestiones de Sociología, 18 e055. https://doi.org/10.24215/23468904e055 


\section{Marcando la cancha: una aproximación al fútbol femenino desde las ciencias sociales}

\section{Drawing the line: A Review of Research on Women's Football}

Martin Alvarez Litke

CONICET, Argentina

Instituto Interdisciplinario de Estudios de Género

(Universidad de Buenos Aires) - CONICET, Argentina

esnowel@hotmail.com

\section{Resumen:}

Si bien el género ha estado presente en los estudios sobre deporte en Argentina desde sus comienzos, aún son escasos los trabajos que analizan la participación de las mujeres en el deporte más importante del país: el fútbol. El presente artículo pretende ser una contribución en este aspecto, analizando la incipiente bibliografía local sobre el fútbol femenino, a la luz de la más amplia producción internacional sobre la temática, mostrando los principales hallazgos, silencios, y las preguntas que quedan pendientes. Recuperamos los aportes de las investigaciones en torno a la sexualidad y la corporalidad de las deportistas, atendiendo a las posibilidades de rearticulación del dispositivo sexo-género-deseo a partir de la práctica del fútbol, y proponemos la necesidad de realizar etnografías que atiendan a la construcción identitaria de las jugadoras y a los sentidos que adquiere su práctica.

Palabras clave: Deporte, Fútbol, Mujeres, Género, Argentina.

\section{Abstract:}

Although the subject of gender has been present in sports studies in Argentina from the start, there are still very few studies that analyze the participation of women in the most important sport in the country: football. This article aims to be a contribution in this aspect, analyzing the incipient local bibliography on women's football, in light of the more extended international production on the subject, showing the main findings, silences, and the questions that remain pending. We recover the contributions of research on the sexuality and corporality of the athletes, taking into account the possibilities of rearticulation of the sex-genderdesire order that derive from the practice of football, and propose the need to carry out ethnographic research on the identitary construction of the women players and the meanings their practice acquires.

KEYWORDS: Sport, Football, Women, Gender, Argentina.

\section{INTRODUCCIÓN}

El género como objeto de estudio y perspectiva de análisis ha estado presente en los estudios sobre deporte en la Argentina desde el primer momento, a partir de los trabajos del fundador del campo, Eduardo Archetti, quien se interesó en la construcción de masculinidades a través de la práctica deportiva y sus relatos mediáticos (1985, 2001 y 2003). El análisis de las masculinidades también ha sido uno de los principales intereses de los estudios sobre las hinchadas de fútbol (Alabarces et al, 2005; Alabarces y Garriga Zucal, 2008). Ahora bien, la relación de las mujeres con el deporte, y en particular con el fútbol, ha sido prácticamente desatendida. De hecho, si revisamos las compilaciones de estudios sobre deporte que han sido publicadas en la Argentina en los últimos tiempos (Alabarces, 2000 y 2003; Branz, Garriga Zucal y Moreira, 2013; Levoratti y Moreira, 2016), de los cuarenta y ocho capítulos que integran las contribuciones de los distintos autores, sólo cuatro focalizan el lugar de las mujeres en el deporte, y reproducen en la academia un sentido común extendido que indica que los deportes son "cosa de hombres".

Justamente, el fútbol ha sido construido como un mundo exclusivamente masculino (Archetti, 1985), y si bien un puñado de autoras ha estudiado el ingreso de las mujeres a este campo pensándolas como espectadoras de partidos (Binello y Domino, 1998; Binello, Conde, Martínez y Rodríguez, 2000; Conde, 2008) o como "mujeres de futbolistas" (Binello y Domino, 1998), no lo han hecho en su rol de jugadoras. Los trabajos 
de Adolfina Janson (2008) y Juan Branz (2012), que examinaremos en profundidad, son los primeros que introducen esta discusión en sus análisis.

El fútbol femenino ${ }^{1}$ ha crecido considerablemente respecto a su número de participantes en los últimos años en la Argentina, hasta llegar a disputar la exclusividad de la práctica a los hombres. En este contexto, el presente trabajo pretende ser una contribución al estudio de este fenómeno a partir del análisis de la literatura académica existente sobre jugadoras de fútbol. A nivel local, resaltamos la escasez de estudios y la falta de análisis con perspectiva de género. En el plano internacional, recuperamos los aportes en torno a la sexualidad y a la corporalidad de las deportistas, poniendo el foco en las posibilidades de rearticulación del dispositivo sexo-género-deseo a partir de la práctica del fútbol.

\section{Estudios sobre JUgAdoras de FÚtbol EN Argentina}

Hasta épocas recientes, el fútbol femenino en la Argentina ha estado invisibilizado, por lo cual no sorprende que los estudios sobre mujeres jugadoras de fútbol aún sean muy incipientes. Pocos autores han incursionado en esta temática, aunque es de esperar que esta tendencia cambie en los próximos años, dado el creciente número de participantes y la visibilidad que está adquiriendo este deporte.

La primera aproximación al estudio del fútbol femenino en la Argentina corresponde a Adolfina Janson (1998 y 2008). La mayor virtud de esta autora ha sido darles voz a las jugadoras publicando sus entrevistas con ellas. Janson distingue dos etapas en la historia del fútbol femenino de nuestro país: la etapa del "jugar por jugar” (2008, p. 239), que se caracteriza por tratarse de una práctica informal, lúdica, de juego por placer; y la etapa del "jugar de veras" (239), que comienza en 1990 con la oficialización del fútbol femenino por parte de la FIFA, lo que pondría fin a la modalidad anterior. En la actualidad, si atendemos a la explosión de experiencias de fútbol recreativo jugado por mujeres en la Ciudad de Buenos Aires y en el resto de la Argentina, parecería más correcto afirmar que las dos lógicas conviven y no que una reemplaza a la otra. La dimensión lúdica persiste, como de hecho también ocurre en el fútbol masculino, incluso en un momento de extrema mercantilización del mismo (Alabarces, 2000).

Janson señala la necesidad -que compartimos- de profundizar la investigación desde una perspectiva de género (1998, p. 208), pero su trabajo deja entrever algunas prenociones que atentan contra este propósito. Al comparar la práctica del fútbol femenino con la del masculino, Janson expresa los "lógicos matices" (206) que diferencian la primera de la segunda: “a) La necesidad de imponer tiempos de juego más cortos (de 25 minutos); b) Respetar el arbitraje (aunque de esto no se puede estar tan segura después de presenciar algunos encuentros); c) Cuidarse el pecho. Cuidar la distancia. Evitar los golpes del adversario" (206).

En primer lugar, debemos alertar sobre el peligro de esta comparación, ya que si bien puede ser útil para mostrar la desigualdad de condiciones en que se lleva adelante este deporte, se corre el riesgo de tomar al fútbol masculino como el parámetro a partir del cual se juzga el fútbol femenino, y definir a este último no desde su especificidad sino a partir de sus carencias y su "diferencia". Por otro lado, coincidimos con Branz (2008) en señalar que las afirmaciones de Janson "nos estarían sugiriendo la vuelta (o la continuidad) a las funciones y/o atributos normativos y prescriptivos de "lo masculino y lo femenino" (49). La autora reproduce los estereotipos masculinos y femeninos reafirmando la superioridad masculina a partir de justificaciones basadas en última instancia en diferencias biológicas que se presentan como dadas. Las diferencias sexuales no son cuestionadas ni problematizadas, sino que se interpretan como "lógicas", lo que sirve nuevamente para justificar una práctica deportiva subalterna y subordinada de las mujeres.

De hecho, un breve análisis del fútbol femenino tal como se practica en la actualidad contradice las afirmaciones de Janson. Respecto del tiempo de juego, es exactamente el mismo en los partidos de hombres que en los de mujeres, tal como lo expresa el Reglamento del Campeonato de Fútbol Femenino de la Asociación de Fútbol Argentino (AFA) ${ }^{2}$. Esto demuestra que si en algún momento existieron diferencias en las reglas del fútbol masculino y el femenino, éstas no respondían a la lógica sino más bien a ideas 
preconcebidas sobre diferencias en las capacidades físicas de hombres y mujeres. De la misma forma, la necesidad de "cuidarse el pecho" (Janson, 1998, p. 206) parece evocar los antiguos argumentos de protección de la función materna utilizados por los detractores de la práctica deportiva de las mujeres (Hargreaves, 1993; Rial, 2013). De hecho, las mismas jugadoras entrevistadas por Janson (2008) desestiman esta preocupación al considerar que nace de un desconocimiento de la técnica del fútbol, ya que para detener el balón con el pecho, se debe recibir la pelota a la altura del esternón y no de los senos (103 y 129).

Por último, la misma autora reconoce en la cita mencionada sus reparos respecto de la premisa de "respeto al arbitraje”, a partir de su propia experiencia como espectadora de partidos entre mujeres. En lugar de dar este tipo de afirmaciones por sentadas correspondería un análisis minucioso de las múltiples relaciones que pueden establecerse entre jugadoras y árbitros o árbitras. En este sentido, Meân (2001) realizó una investigación sobre el arbitraje en Inglaterra y describió las sutiles prácticas discursivas a través de las cuales los referís hombres socavan la legitimidad de los reclamos realizados por jugadoras mujeres, mediante actitudes paternalistas que presuponen un desconocimiento de las reglas de juego por parte de las jugadoras.

El fútbol femenino en la Argentina también ha sido analizado por Juan Branz, cuya mirada es doblemente interesante por enfocarse desde los estudios culturales pero también desde su condición de exjugador de fútbol. Branz (2012) realizó un estudio etnográfico en el cual participó como entrenador de un grupo de mujeres de clase media en una plaza pública de la ciudad de La Plata. A partir de esta experiencia, el autor pudo comprobar que al practicar fútbol en público las mujeres se exponen a la mirada sexualizante de los hombres, quienes a través de sus comentarios machistas minimizan el valor de la práctica deportiva de las mujeres y ponen el foco en la erotización de sus cuerpos y movimientos (346). Es significativo, entonces, que en el transcurso de su experiencia etnográfica, las mujeres trasladaran su práctica de la plaza -pública y gratuita-, a una cancha de Fútbol 5 -privada y arancelada-, "de la exposición a la reclusión" (349). En este sentido, Branz sostiene que el circuito comercial acompaña la iniciativa de estas mujeres y la mantiene en el ámbito privado, lo que al mismo tiempo opera una exclusión, ya que quedan fuera quienes no cuentan con los recursos para acceder a este circuito arancelado (350).

Por otro lado, Branz comprobó que las jugadoras a través de sus discursos y prácticas tendían a "masculinizar los modos de nombrar sus destrezas, sus saberes y sus intenciones lúdicas" (346), por lo cual concluyó que no estarían disputando el sentido que los hombres dan al fútbol sino más bien reproduciendo sus lógicas y sus retóricas. De manera que, para él, la práctica de fútbol por parte de las mujeres podría "no estar dada como una reivindicación de género, sino como una distinción en referencia a otras mujeres” (350). Esta afirmación es interesante, pero sería necesario atender a las representaciones que las jugadoras de estos circuitos privados tienen de su propia practica, para dilucidar si esa "distinción" es parte de una reivindicación identitaria o si es más bien una consecuencia indirecta de la expulsión del espacio público. Éste no es un detalle menor, ya que la dificultad de acceso a los espacios públicos ha sido identificada como uno de los mayores obstáculos para la práctica deportiva de las mujeres a nivel mundial (Hartmann-Tews y Pfister, 2005).

\section{EL FÚTBOL FEMENINO EN OTROS PAÍSES}

Como podemos ver, la producción académica sobre jugadoras de fútbol en Argentina ha sido realmente escasa hasta el momento, y si bien los trabajos mencionados proponen algunas líneas de investigación interesantes, es necesario realizar estudios etnográficos en profundidad para comprender el fenómeno del fútbol femenino en toda su complejidad y en sus múltiples dimensiones de análisis. En este sentido, vale la pena retomar la producción académica internacional sobre jugadoras de fútbol, ya que introduce problemáticas que no han sido consideradas seriamente en el contexto local. Nos referimos a las temáticas predominantes en los estudios etnográficos sobre el fútbol femenino: la corporalidad y la sexualidad. Los autores han prestado atención al estereotipo que pesa sobre el fútbol femenino respecto del lesbianismo -lo que ha llevado a algunos a 
hablar del "problema de la imagen del fútbol femenino" (Harris, 2005)-, pero también han analizado y problematizado la presencia real de lesbianismo en el fútbol jugado por mujeres (Cauldwell, 2002, p. 30).

Así, Mennesson y Clément (2003) intentan explicar la fuerte presencia del lesbianismo en el fútbol femenino en Francia. Sostienen que "la homosociabilidad en los equipos de fútbol de mujeres autoriza y facilita las prácticas homosexuales" (320 - traducción propia), y permite a las jugadoras que así lo deseasen tomar los pasos decisivos para involucrarse en este tipo de prácticas sexuales (320). Sin embargo, sostienen que la homosociabilidad no puede explicar por sí sola la emergencia de prácticas homosexuales, ya que en los equipos masculinos esta sociabilidad intragenérica coincide con un fuerte rechazo hacia la homosexualidad (321). Más bien, la homosociabilidad forma parte de una combinación de factores que influyen en la sexualidad de las jugadoras, entre los cuales incluyen la experiencia personal de cada una de ellas y el hecho de que el fútbol esté fuertemente asociado a la masculinidad (322), lo cual "favorece la adopción de una posición crítica respecto de las normas de género dominantes” (328 - traducción propia).

Respecto a este tema, Cauldwell (2002) sostiene que en determinados contextos la visibilidad del lesbianismo en el fútbol "desestabiliza, subvierte y resiste la construcción del espacio heterosexual" (35 traducción propia).Así, introduce el concepto de dykescape (35), para denotar la invasión y apropiación de un espacio heterosexual por parte de mujeres lesbianas. Los dykescapes ofrecen a las lesbianas el placer del reconocimiento denegado en otras áreas. Se trata de desafíos a la heterosexualidad dominante que son negociados y momentáneos: "esta subversión y transformación del espacio representa momentos en los cuales las prácticas regulatorias utilizadas para proteger la heterosexualidad y reforzar la heteronormatividad son neutralizadas" (41 - traducción propia). La autora sostiene que la demarcación del fútbol femenino como lésbico genera tensión para las jugadoras heterosexuales (34), que experimentan una marginación en determinadas culturas futbolísticas, lo cual pone de manifiesto la fragilidad de la heterosexualidad obligatoria. Estos espacios de visibilidad lésbica permiten que la posición privilegiada de la heterosexualidad sea cuestionada y repensada (39).

Cox y Thompson (2000 y 2001), por su parte, han señalado que en su práctica deportiva las jugadoras de fútbol se enfrentan con discursos contradictorios sobre sus cuerpos. El discurso deportivo postula que el cuerpo debe ser físicamente poderoso, mientras que el discurso heteronormativo construye el cuerpo femenino como débil y pasivo en contraposición al cuerpo masculino, fuerte y activo (Cox y Thompson, 2000, p. 8). La lógica binaria y contradictoria de estos discursos implica que el ideal deportivo está en sintonía con el ideal masculino, pero se opone al ideal femenino. Esta contradicción se acentúa aún más cuando el deporte en cuestión ha sido asociado tradicionalmente a los hombres, como es el caso del fútbol. Por lo tanto, jugar al fútbol implica para las mujeres un desafío a las fronteras de género y a la heterosexualidad (Cox y Thompson, 2001, p. 9). En este sentido, Cauldwell (2003) plantea que las corporalidades deportivas de las mujeres pueden desafiar el orden establecido de la heterosexualidad obligatoria y crear rearticulaciones del dispositivo sexo-género-deseo. La masculinidad femenina y el lesbianismo en el deporte pueden romper con el orden obligatorio mujer-femenina-heterosexual, lo que podría traer aparejadas nuevas posibilidades identitarias (Cauldwell, 2002, p. 383).

Ahora bien, estos procesos son complejos, y las futbolistas continuamente deben negociar con los discursos contradictorios del deporte, el género y la heterosexualidad, desafiándolos y reforzándolos al mismo tiempo (Cox y Thompson, 2000, p. 17). En un estudio etnográfico con jugadoras de fútbol en Nueva Zelanda, Cox y Thompson (2000 y 2001) encontraron que las deportistas se mostraban satisfechas con las capacidades físicas que desarrollaban a partir de su práctica futbolística, pero al mismo tiempo estaban frustradas por no adecuarse al modelo de cuerpo femenino heterosexualmente deseable. Las jugadoras vigilaban su imagen para adecuarla a las construcciones dominantes de femineidad (Cox y Thompson, 2001, p. 21), utilizando el cabello largo para diferenciarse no sólo de los hombres, sino también de la imagen estereotípica de la lesbiana de pelo corto (2000, p. 14). 
Además, las jugadoras heterosexuales trataban de disociarse de sus compañeras lesbianas (2001, p. 21). Harris (2005) observó prácticas similares en su investigación en Inglaterra, pero sugiere que estas actitudes podrían relacionarse con la experiencia de las mujeres heterosexuales en "ambientes futbolísticos donde las depredadoras sexuales están a sus anchas" (194 - traducción propia). Aquí el autor incurre en el estereotipo homofóbico de la lesbiana como depredadora (Cauldwell, 2002; Cox y Thompson, 2001), según el cual se asume que la mirada lésbica necesariamente expresa un deseo sexual indiscriminado y una atracción hacia cualquier cuerpo de mujer (Cox y Thompson, 2001, p. 17). Este estereotipo es particularmente nocivo en un contexto en el cual las jugadoras comparten vestuarios donde sus cuerpos están expuestos a la mirada de otras mujeres. En este sentido, la construcción homofóbica de las lesbianas como el "cuco" $\underline{3}$ (2001) del fútbol femenino perjudica al deporte y presiona a todas las jugadoras, heterosexuales y homosexuales por igual, a presentar una imagen de femineidad heterosexual (Cox y Thompson, 2000, p. 16).

\section{COMENTARIOS FINALES}

Las investigaciones citadas a lo largo de este artículo presentan múltiples aristas a partir de las cuales analizar el fútbol femenino y las formas en que la participación de las mujeres en este deporte entra en tensión con las representaciones, prácticas y relaciones sociales dominantes de género, sexualidad y deporte. En la Argentina los avances en esta dirección han sido escasos, y se impone como necesidad llevar adelante investigaciones etnográficas que den cuenta de la manera en que estas relaciones se ponen en juego en el contexto local para configurar las identidades de las jugadoras.

En un país en el cual el fútbol constituye una parte esencial del imaginario nacional, la subordinación e invisibilización histórica de las mujeres en este deporte representa -como en Brasil (Rial, 2013)- una exclusión de la participación plena en la nación. Si atendemos a esto, el crecimiento reciente del fútbol femenino puede animarnos a establecer apresuradamente su carácter contrahegemónico, pero es necesario analizar si esta práctica está creando nuevos significados o si, por el contrario, reproduce las lógicas masculinas de un deporte pensado por y para los hombres. El sentido del fútbol, como el de cualquier deporte, no está dado de una vez y para siempre; es motivo de luchas por la imposición de su definición legítima (Bourdieu, 1990). Para dilucidar estos sentidos es necesario que cualquier investigación etnográfica sobre el fútbol femenino tenga en cuenta las relaciones de poder que operan en el campo, para entender de qué manera las lógicas son reproducidas, subvertidas, o negociadas.

\section{REFERENCIAS}

Alabarces, P. (Comp.). (2000). Peligro de Gol. Estudios sobre deporte y sociedad en América Latina. Buenos Aires: CLACSO.

Alabarces, P. (Comp.). (2003). Futbologías. Fútbol, identidady violencia en América Latina. Buenos Aires: CLACSO.

Alabarces, P., Conde, M., Dodaro, C., Fernández, F., Ferreiro, J. P., Galvani, M.,... Salerno, D. (2005). Hinchadas. Buenos Aires: Prometeo Libros.

Alabarces, P. y Garriga Zucal, J. (2008). El “aguante”: una identidad corporal y popular. Intersecciones en antropología, 9, 275-289. Recuperado de http://www.scielo.org.ar/scielo.php?script=sci_arttext\&pid=S1850-373X200800 0100020

Archetti, E. (1985). Fútbol y ethos. Monografias e informes de investigación, 7, 71-109.

Archetti, E. (2001). El potrero, la pista y el ring. Las patrias del deporte argentino. Buenos Aires: Fondo de Cultura Económica.

Archetti, E. (2003). Masculinidades: fútbol, tango y polo en la Argentina. Buenos Aires: Antropofagia. 
Binello, G., Conde, M., Martinez, A. y Rodriguez, M. G. (2000). Mujeres y fútbol: ¿̇territorio conquistado o a conquistar?. En P. Alabarces (Comp.), Peligro de Gol. Estudios sobre deporte y sociedad en América Latina (pp. 33-55). Buenos Aires: CLACSO.

Binello, G. y Domino, M. (1998). Mujeres en el área chica. En P. Alabarces et al. (Eds.), Deporte y sociedad (pp. 211-226). Buenos Aires: Eudeba.

Bourdieu, P. (1990). ¿Cómo se puede ser deportista? En Sociología y cultura (pp. 142-158). México: Grijalbo.

Branz, J., Garriga Zucal, J. y Moreira, V. (Comps.). (2013). Deporte y Ciencias Sociales: claves para pensar las sociedades contemporáneas. La Plata: Edulp.

Branz, J. (2008). Las mujeres, el fútbol y el deseo de la disputa: cuando lo deportivo debe volverse político. Educación Física y Ciencia, 14, 45-57. Recuperado de http://www.memoria.fahce.unlp.edu.ar/art_revistas/pr.3696/pr.36 96.pdf

Branz, J. (2012). Fútbol, mujeres y espacio público. En G. Cachorro (Comp.), Ciudad y prácticas corporales (pp. 339-352). La Plata: Edulp.

Caudwell, J. (2002) Women's experiences of sexuality within football contexts: A particular and located footballing epistemology. Football Studies 5(1), 24-45. Recuperado de http://library.la84.org/SportsLibrary/FootballStud ies/2002/FS0501e.pdf

Caudwell, J. (2003). Sporting Gender: Women's Footballing Bodies as SitesSights for the (Re)Articulation of Sex, Gender, and Desire. Sociology of Sport Journal 20(4), 371-386. doi: https://doi.org/10.1123/ssj.20.4.371

Conde, M. (2008). El poder de la razón: las mujeres en el fútbol. Nueva sociedad 218, 122-130. Recuperado de http: //nuso.org/media/articles/downloads/3575_1.pdf

Cox, B. y Thompson, S. (2000). Multiple Bodies. Sportswomen, Soccer and Sexuality. International Review for the Sociology of Sport 35(1), 5-20. doi: https://doi.org/10.1177/101269000035001001

Cox, B. y Thompson, S. (2001). Facing the Bogey: Women, Football and Sexuality. Football Studies 4(2), 7-24. Recuperado de http://library.la84.org/SportsLibrary/FootballStudies/2001/FS0402d.pdf

Hargreaves, J. (1993). Promesa y problemas en el ocio y los deportes femeninos. En J. L. Barbero et al. (Eds.), Materiales de Sociologia del Deporte (pp. 109-132). Madrid: La Piqueta,.

Hargreaves, J. (2003). Sporting females. Critical issues in the history and sociology of women's sports. Londres: Routledge.

Harris, J. (2005). The image problem in women's football. Journal of Sport and Social Issues 29(2), 184-197. doi: htt ps://doi.org/10.1177/0193723504273120

Hartmann-Tews, I. y Pfister, G. (2005). Sport and women. Social issues in international perspective. Londres: Routledge.

Janson, A. (1998). Aproximaciones al tema del fútbol femenino y los límites a tener en cuenta para una interpretación sociológica. En P. Alabarces et al. (Comps.), Deporte y sociedad (pp. 203-210). Buenos Aires: Eudeba.,

Janson, A. (2008). Se acabó este juego que te hacía feliz. Nuestro fútbol femenino (desde su ingreso a la AFA en 1990, hasta el Mundial de Estados Unidos en 2003). Buenos Aires: Aurelia Rivera Grupo Editorial.

Levoratti, A. y Moreira, V. (Comps.). (2016). Deporte, cultura y sociedad. Estudios socio-antropológicos en Argentina. Buenos Aires: Teseo.

Meân, L. (2001). Identity and discursive practice: doing gender on the football pitch. Discourse and Society 12(6), 789-815. doi: https://doi.org/10.1177/0957926501012006004

Mennesson, C. y Clément, J. (2003). Homosociability and Homosexuality. The Case of Soccer Played by Women. International Review for the Sociology of Sport 38(3), 311-330. doi: https://doi.org/10.1177/10126902030383 004

Rial, C. (2013). El invisible (y victorioso) fútbol practicado por mujeres en Brasil. Nueva Sociedad 248, 114-126. Recuperado de http://nuso.org/media/articles/downloads/3995_1.pdf 


\section{Notas}

1 Utilizaremos prioritariamente fútbol femenino para referirnos a la práctica de este deporte por parte de las mujeres, ya que de esa forma se lo nombra y reconoce en la Argentina. No obstante, aclaramos que se trata del mismo juego, con las mismas reglas, sea practicado por hombres o mujeres (Rial, 2013).

2 "Los partidos tendrán la duración de noventa (90) minutos en dos períodos de cuarenta y cinco (45) minutos cada uno, con un intervalo de quince (15) minutos" (Boletín Especial № 5250 de la Asociación de Fútbol Argentino - Reglamento del Campeonato de Fútbol Femenino Temporada 2016/2017)

3 Cox y Thompson (2001) utilizan el término bogey, que hemos traducido como "cuco", para denotar el miedo irracional que genera la presencia de lesbianas en el fútbol femenino. 